\title{
La universidad peruana y el desarrollo nacional: conflictos y posibilidades
}

\section{Peruvian university and national development: conflict and posibilities}

\author{
Edward Faustino Loayza Maturrano ${ }^{1}$ \\ "La educación en el Perú no tiene un espíritu nacional; por \\ el contrario, es una educación colonial y colonizadora." \\ José Carlos Mariátegui
}

\begin{abstract}
RESUMEN
El presente ensayo expositivo-argumentativo pretende revisar la problemática del sistema universitario peruano, sus diversas relaciones dentro de la sociedad, así como su justificada importancia para el desarrollo del país, adoptando para ello una perspectiva crítica y propositiva. Por tal razón, se analizan los aspectos problemáticos de la Universidad, tales como 'la concepción de universidad', 'política universitaria', 'gestión institucional', 'investigación y generación del conocimiento' y la 'vinculación con la sociedad'.
\end{abstract}

Palabras clave: universidad; multiversidad; heteronomía universitaria; modelo universitario; política universitaria; revolución académica; innovación y reforma universitaria.

\begin{abstract}
The present expositivo-argumentative rehearsal seeks to revise the problem of the peruvian university system, its diverse relationships inside the society, as well as its justified importance for the development of the country; adopting for it a critical perspective and of proposal. For such a reason, the problematic aspects of the University are analyzed, such as 'the university conception', 'university politics', 'institutional administration', 'investigation and generation of the knowledge' and the 'linking with the society.'
\end{abstract}

Keywords: university; multiversity; university heteronomy; university model; university politicsm; academic revolution; innovation and reformation university student.

\section{INTRODUCCIÓN}

A través del tiempo, en la sociedad peruana han existido y existen ámbitos problemáticos que, aún en la actualidad, no han podido resolverse abarcadoramente. Uno de esos ámbitos está referido a la educación superior y, dentro de esta, primordialmente, la educación universitaria.
Una primera labor fundamental para abordar la cuestión es detectar los principales aspectos problemáticos involucrados en la educación universitaria; esto es, efectuar la diagnosis ${ }^{1}$. En tal sentido, caracterizando la situacionalidad encontramos que la 'concepción de universidad', la 'política universitaria', la 'gestión institucional', la 'investigación y generación del

\footnotetext{
${ }^{1}$ Departamento de Ciencias Humanas, Facultad de Economía y Planificación, Universidad Nacional Agraria La Molina, Lima, Perú. Email: edwloma@lamolina.edu.pe
} 
conocimiento', y su 'vinculación con la sociedad' son unidades de análisis. En cada uno de estos aspectos problemáticos aún no existen respuestas lo suficientemente válidas. No se han determinado el qué, el para qué, el por qué ni mucho menos el con qué, el dónde, el cuánto, el cómo, el cuándo del empleo de un modelo peruano de sistema universitario enmarcado dentro de la ejecución de un plan de desarrollo educativo nacional diversificado.

Las exigencias de una educación de calidad, que se sustente cada vez más en los avances de la ciencia y la tecnología, y que responda a los cambios de la sociedad y de sus nuevos retos es una necesidad imperiosa de nuestros tiempos. De allí que se descubra, cual imponente iceberg, por ser señera cúspide de toda pirámide socioeducativa: la Universidad.

\section{ANÁLISIS DEL ESTADO DE LA CUESTIÓN}

Universidad, concepto que aglutina al universo y a la verdad en uno. Ella significa unidad, coherencia, y adecuación del conocimiento, y del saber de toda la humanidad, siempre tras los pasos de las nuevas interrogantes veritacionales del hombre y de su situación cultural que constantemente se renueva.

Esta simbiosis de la artificialidad del hombre, la ciencia; le da luz y conduce al invidente humano hacia su progreso evolutivo. La Universidad, institución donde, históricamente, se genera y acumula el conocimiento científico, es en esencia, la madre del saber humano. Esta condición adquirida histórica y culturalmente es el leit motiv por el cual reviste la preocupación central del ser humano en su afán de promover el desarrollo de la humanidad mediante políticas de desarrollo de la Verdad, en primer lugar, y, en segundo, a través de políticas de Estado que hagan posible el avance científico dentro de una sociedad como la nuestra.

En la sociedad, la educación universitaria está representada institucionalmente por la Universidad. Esta, sin embargo, en el Perú, desde sus albores, ha tenido como brújula la cerrazón de la tradición escolástica, cuya base teológica lacró las posibilidades de la especulación por la transmisión de una cultura filosófica mística (modelo de universidad medieval). Bajo este modelo colonial ${ }^{2}$ en 1551 se crearon las universidades de San Marcos en Lima y, algunos años después, de la Real y Pontificia Universidad de México cuando todavía no había ninguna en toda América.

La universidad, entonces, tuvo un carácter unitario en su concepción y propósito definido dentro de la estructura académica que se encontraba al servicio de la monarquía. Estos rasgos vinculan al modelo universitario colonial con la tradición de la universidad de Salamanca, que, a su vez, incorpora rasgos propios de la 'universidad de estudiantes' (universitas scholarium) del modelo de Bolonia ${ }^{3}$; que tiene su contrapartida en el modelo de 'universidad de profesores' (universitas magistrorum) de la universidad de París ${ }^{4}$.

En el Perú republicano, la Universidad se estructuró de acuerdo con el denominado modelo napoleónico ${ }^{5}$ con lo que se pierde el modelo de universidad como 'institución universitaria', característica de la universidad del colonialismo. Asimismo, este modelo emergente de la universidad republicana peruana consolidó otra característica de la universidad colonial: la entera lejanía de la ciencia. Otra circunstancia adversa al desarrollo de la universidad republicana es que, en nuestro país no prosperaron las academias e institutos que en Francia asumieron la tarea de promover el adelanto científico.

Por otra parte, la proximidad de la Universidad con el poder, es decir, la intensa politización de los estudiantes y profesores 
universitarios, hizo que se formaran élites y contraélites. Ello, consecuentemente, produjo el choque entre élites gubernamentales por el poder. Así, la universidad republicana se convirtió en oligarquía, esto es, de base social muy reducida en cuanto al acceso. La universidad republicana se transformó en la 'universidad-nación', o mejor dicho, en el 'patriarcado universitario' (hijos de hacendados, funcionarios, etc., quienes eran formados para el desempeño de cargos político-burocráticos y de mantenimiento del orden social puestos al servicio de la clase dominante).

A finales de la segunda década del siglo XX, los estudiantes de San Marcos en el Primer Congreso Nacional de Estudiantes reunidos en Cusco acogieron el ideario de la reforma de los estudiantes cordobeses $^{6}$, adoptando una resolución de gran trascendencia: la creación de las "universidades populares González Prada", uno de los aportes de la primera reforma universitaria peruana. En estos centros confraternizaron obreros, estudiantes e intelectuales, ampliándose de ese modo el radio de influencia de la reforma. El reformismo peruano, en efecto, aparece como más politizado, siendo una expresión de este la fundación del APRA.

Esta reforma desconoció, al propio tiempo, que en Europa del siglo XIX se había producido una gran transformación de la universidad, considerada por muchos como una revolución académica, el surgimiento de la 'universidad investigación' de Alemania. En ese entonces, la nación germánica se había convertido en el gran teatro del avance científico, debido a la interacción fecunda entre investigación y enseñanza superior, es decir, a la combinación de fuerzas, presencia y colaboración de alumnos en la tarea de investigar.

El progreso de la ciencia es, manifiestamente, más rápido y más vivo en una universidad donde se desarrolla constantemente, y, además, a cargo de un número de cabezas rigurosas, lozanas y juveniles. La ciencia no puede nunca exponerse verdaderamente como tal, ciencia, sin empezar por asimilársela independientemente, $\mathrm{y}$, en estas condiciones, no sería concebible que, de vez en cuando, e incluso frecuentemente, no hiciese algún descubrimiento (Schwartzman, 1996: 33).

Por todo, esto se desprende que el modelo alemán posibilitó el sólido impulso a la investigación determinando el avance del conocimiento científico aunque desligado de los requerimientos sociales. Lo que al decir de Wilhelm Von Humboldt ${ }^{7}$ es la unidad entre investigación, docencia y estudio. Las universidades alemanas y aquellas europeas que siguieron este modelo alcanzaron nuevos y relevantes conocimientos científicos en las primeras décadas del siglo XX.

Este modelo alternativo, el germánico -es menester señalar-, no se implantó en el país ni durante el siglo XIX ni aun en el siglo XX, puesto que la politización desligó con fortaleza a la universidad peruana de su rol científico.

A lo largo de la segunda mitad del siglo $\mathrm{XX}$, sin embargo, se implantó un modelo "desarrollista" de universidad, el cual es y sigue siendo un prototipo asociante entre el napoleónico y el norteamericano ${ }^{8}$, que plantea la producción en serie de titulados de acuerdo a las necesidades del mercado (regidas por las curvas de la oferta y la demanda); enfoque que niega rotundamente la necesidad de la investigación científica seria, consolida el atraso y la pobreza de una nación, minimizando o estancando cualquier afán de desarrollo.

La Universidad de acuerdo a este formato francés está constituida por facultades tradicionales, diseñadas para la formación de profesionales liberales que aglutinan la mayor parte de la población estudiantil universitaria. Si bien su impacto no constituye en sí misma un núcleo 
transformador de la vida universitaria que supere el estado de marginalidad de las actividades de investigación y dé una nueva dinámica científica más allá de los ámbitos de las profesiones tradicionales, esta se configura en un primer intento de construcción de modelos de organización universitaria alternativa. Su debilidad más grande es ser heredera del espíritu humanista que concibe lo académico como una opción de vida de algunos talentos escogidos, como una actividad espontánea, subsidiaria, en sí misma "honorífica", y que está ausente en el sentido de la actividad profesional.

Construir un ideal de Universidad posible para el Perú, más acorde con las exigencias de nuestro entorno y la cultura propia, requiere reconocer y preguntarnos por la historia y los modelos de universidad que hoy existen en el mundo, premisa que se asume como válida, y, a partir de la cual, se ha de configurar el modelo de universidad en, por y del Perú con el fin de hacer posible una mejor sociedad dentro de una nueva mentalidad científica, por urgente y necesaria.

En el aspecto académico, se han producido visiblemente transformaciones en el entorno que están afectando la estructura y el desenvolvimiento de las instituciones universitarias. Esto hace referencia a los contextos de acceso a la información, de manejo del conocimiento, y de la investigación y producción científica.

Es sabido que a lo largo de la historia, la información ha sido escasa y de difícil acceso. Desde la invención de la escritura, la más rica información estuvo depositada en textos que eran accesibles solo para una pequeña minoría incluso hasta entrado el siglo XIX, cuando todavía el analfabetismo era alto alrededor del mundo.

Recién con la imprenta se produjo una verdadera revolución, al quedar la escritura registrada en textos de fácil reproducción. Pero, también, el libro y los periódicos demoraron en masificarse y solo en las últimas décadas experimentaron una explosión. Así, por ejemplo, mientras la Biblioteca de la Universidad de Harvard demoró 275 años en reunir su primer millón de libros, el último lo reunió en solo 5 años.

Hoy el cuadro es completamente distinto; la información disponible es cada vez más abundante y fácil de obtener, como lo muestran el Internet y la World Wide Web (WWW). En la actualidad las páginas de Internet están creciendo a razón de dos millones por día. Se constata, asimismo, que entre los primeros en usar las tecnologías de red con fines científicos se encuentran los estudiantes de educación superior, los profesionales y técnicos superiores $(C f$. Brunner, 2000).

Por tanto, en la actualidad, el problema no es dónde encontrar la información, sino cómo establecer acceso a ella sin exclusiones y a la vez, cómo enseñar y aprender a seleccionarla, evaluarla, interpretarla, clasificarla y usarla. Una consecuencia de esta verdadera revolución, es que las instituciones formales de educación dejan de ser el principal medio de información y deben competir con otros medios como la televisión e Internet, de cuyo uso se espera también, información y enseñanza.

Una cuestión adicional es cómo la sociedad —desde la escuela hasta la universidad- desarrolla esas funciones que la psicología contemporánea denomina funciones cognitivas superiores, tales como "identificación y solución de problemas, planificación, reflexión, creatividad, comprensión en profundidad" (Gardner, 1999), indispensables en un medio saturado de información.

Un segundo contexto que está cambiando rápidamente es el del manejo del conocimiento, que es la materia con la que trabajan las universidades. Hasta hace poco tiempo, la plataforma del conocimiento avanzado era relativamente reducida $\mathrm{y}$ 
estable, lo que facilitaba su transmisión y apropiación.

Hoy, en cambio, el conocimiento aumenta y cambia con gran velocidad. De acuerdo a las mediciones efectuadas por los bibliometristas, demoró 1750 años en duplicarse por primera vez el volumen del conocimiento humano. La siguiente vez lo hizo en 150 años, luego en 50 y hoy se duplica cada cinco años. Se estima que el año 2020 aumentará al doble cada 73 días. Por su parte, las revistas científicas han aumentado de 10 mil en 1900 a más de 100 mil en la actualidad. Los hombres y mujeres sabios de antaño son reemplazados por minuciosos expertos que "publican o perecen" ( $C f$. Appleberry, 1 998).

Esto también puede ilustrarse con ejemplos tomados de algunas disciplinas. En la química, desde comienzos de los años 90, cada 2 años más de 1 millón de artículos aparecen en las revistas especializadas (Clark, 1998). A su turno, entre 1978 y 1988, el número de sustancias conocidas pasó de 360 mil a 720 mil, alcanzando en 1998 a 1.7 millones (Salmi, 2000). En la biología, recién en 1977 se diseñó el método para determinar la secuencia de bases (las letras que codifican la información del ADN), que inicialmente permitía determinar la secuencia de 500 bases por semana. Hoy ese mismo método, perfeccionado y robotizado, permite descifrar los 3000 millones de bases del genoma humano en unos pocos años. Actualmente, un centro de genómica puede determinar un millón de bases por día. En las matemáticas, según un entendido, cada año se dan a conocer más de 100 mil nuevos teoremas. En la historia, la disciplina produjo en dos décadas —entre1960 y 1980 - más publicaciones que en todo el período anterior desde la historiografía clásica de Grecia ( $C f$. Brunner, 2000).

En estas condiciones, ha dicho Howard Gardner (1999:53):

El individuo (o agente inteligente) que pueda examinar esos cuerpos de conocimiento y determinar qué vale la pena conocer, recibirá un significativo premio. Aún más demandada será la persona -o el buscador- capaz de sintetizar esos dominios de conocimiento que crecen esponencialmente, logrando así que información vital esté a disposición de manera útil, para el ciudadano y el formulador de políticas.

En el tercer ámbito, la investigación cumple funciones de variada índole en la universidad. Desde luego, permite un mejor manejo del conocimiento acumulado en las disciplinas sobre las que versa (lo que, a su turno, mejora la docencia de pre y postgrado); pero, además, favorece un ejercicio cotidiano del ethos académico, contribuye a la consolidación de comunidades al interior de la universidad y permite mejorar la posición relativa de la institución -medida en función del desempeño- en el sistema de educación superior.

Si bien resultaría excesivo afirmar que las Universidades son imprescindibles a la hora de hacer investigación (la historia de la ciencia está llena de casos en los que la frontera del conocimiento se movió desde fuera de la universidad y en los hechos, las empresas, los politécnicos y un conjunto de otras instituciones también investigan) es claro que la Universidad no puede desarrollar bien sus tareas si no cuenta con rutinas de investigación.

En nuestro país, tanto la investigación y la producción del conocimiento están desarticuladas de las agendas de políticas de Estado; por lo que la falta de una política integrada para la formación de investigadores, los pocos recursos destinados para la investigación, la poca vinculación inter e intra universitaria en investigación, así como la falta de un sistema de evaluación de los postgrados se constituyen en las características principales de la problemática, más aún si en éstos 
últimos se encuentra la crema de la materia gris de nuestra universidad.

La investigación puede prescindir de la Universidad; pero la Universidad no puede prescindir de la investigación. Por eso, suele afirmarse que está en la misma índole de la Universidad desarrollar esta actividad. La investigación requiere, sin embargo, de financiamiento. La Universidad cuenta con recursos limitados para atender una gran demanda de fondos de investigación de parte de sus profesores. Se hace necesario entonces elegir o, lo que es lo mismo, racionalizar esos recursos para que vayan a sus usos más urgentes definidos por la índole del trabajo universitario y por las metas de pervivencia de la Universidad.

Todo esto presenta serios desafíos para el sistema educacional y la universidad peruana en particular. ¿Quiénes serán mañana los sintetizadores de conocimiento? A la enseñanza de las competencias profesionales: ¿Qué otras es necesario añadir, en qué momento y bajo qué modalidades? ¿Cómo organizar curricularmente un conocimiento en permanente cambio y expansión? A la investigación: ¿En qué áreas prioritarias se ha investigar para lograr conocimientos científicos de acogida mundial?.

Otro aspecto problemático es la política y gestión universitaria, así como la vinculación con las distintas instituciones sociales: Estado, Iglesia, empresa, sociedad civil y cultura principalmente. Al contrario de lo que ocurre en naciones y sistemas más dinámicos, en el Perú, seguimos apegados a un modelo de educación superior introvertido, de transmisión analógica, renuente a la diferenciación y flexibilización, que considera a cada institución aisladamente y no como parte de una red, temeroso de la competencia y con sospechas respecto a la colaboración público-privada.

La relación universidad y sociedad, desde los comienzos del nacimiento de las universidades se constituyó en un elemento fundamental en la determinación de la institucionalidad. La relación entre las universidades, el Estado, la Iglesia y los intereses ha sido siempre una constante, por supuesto, con diferencias en el tipo de relación y en la preeminencia de una y otra. No obstante, desde la Edad Media, las universidades han proporcionado a la sociedad una variedad de servicios utilitarios y formación de sacerdotes, abogados, médicos y funcionarios.

Una universidad sustentada en una autonomía de gestión que adolece del síndrome del autismo, no puede integrarse al ritmo que la dinámica social le exige. Es pues, un ser social sin trascendencia supranacional, o mejor dicho, con incapacidad de internacionalización. El ethos teleológico consustancial a su ser: la búsqueda de la verdad, ha de conducir toda estrategia de política y gestión universitaria. Si bien la universidad nunca ha sido totalmente autónoma, y de hecho ha sido influida anteriormente por fuerzas externas, estamos presenciando un nuevo modelo de subordinación que va más allá del clásico control de una institución por parte de un grupo empresarial o de la intervención coyuntural de un gobierno autoritario. Esto conduce a la sospecha de que la universidad se está volviendo más heterónoma sin que deba entenderse el proceso en términos dicotómicos, como que ha sido despojada, o va a ser despojada en el futuro de toda condición autónoma. Aunque, lo que sí puede pensarse es que el margen de autonomía institucional, básicamente la sustantiva (entendida como el poder que tiene la universidad para determinar sus propios objetivos y programas), se ha ido reduciendo paulatinamente, en razón de que el entorno ${ }^{9}$ y el propio Estado han incrementado su capacidad para imponer su propia lógica e intereses.

Entre los polos de conducción, de un lado, del Estado al sistema universitario 
y, por otro, la absoluta autonomía de estas instituciones, la tendencia internacional en nuestros días es hacia una coordinación intermedia: los países descentralizados (como el Reino Unido o los Estados Unidos), están moviéndose hacia una mayor coordinación pública, mientras que los países muy centralizados (Francia, Alemania), se dirigen hacia una mayor autonomía de las instituciones. En el modelo de coordinación intermedia, el Estado, junto con respetar la autonomía de las instituciones, reclama para sí la función de determinación de prioridades de acciones y de orientación, tanto del sistema como de sus actores, a través de instrumentos regulatorios y de financiamiento.

La función del Estado en el sistema universitario no es la de un espectador, ni la de un benévolo dispensador de recursos, tampoco la de un interventor. El papel del Estado ha pasado a ser más el de un ente regulador y orientador, que un ejecutor único de las tareas. Su misión es la de representar y apoyar el interés nacional en la Educación Superior. La especial relación del Estado con las universidades públicas deriva de esta noción, lo que implica un compromiso recíproco: el de las universidades, de orientar su quehacer hacia el bienestar y desarrollo del país, cada una según su misión particular y el del Estado, de apoyar la capacidad de las instituciones para cumplir con esta orientación. En cuanto a las instituciones privadas, el Gobierno respeta y valora la diversidad de sus misiones, mientras cumplan con sus fines como instituciones de educación y está dispuesto a continuar apoyando las funciones que ellas realizan en beneficio de la prosperidad del país en su conjunto. La gobernabilidad del sistema universitario circunscrita en este tipo de gestión participativa posibilita la rapidez adaptativa frente a los cambios.

Esto último no significa que el modelo heterónomo asuma que la universidad pasa a ser súbitamente gobernada por agentes extrauniversitarios, sino que su práctica cotidiana se encuentra cada vez más supeditada a la lógica del entorno. Obviamente, el peso específico respectivo del Estado y del entorno en la vida universitaria varía en cada uno de las regiones y ciudades, aun más, de institución a institución. En este sentido, es pertinente aclarar que el concepto de heteronomía constituye una categoría abstracta que permite el análisis teórico de la reestructuración universitaria, hoy en día, pero su valor explicativo y su uso pragmático dependerá de cada contexto y de la formación de sus intérpretes.

Desde el punto de vista conceptual, lo que la categoría de universidad heterónoma permite es condensar, bajo un solo término, dos tendencias paralelas que en general son examinadas en forma separada en los estudios de educación superior: la universidad comercial y la universidad controlada. Por lo que el concepto de universidad heterónoma desafía la concepción de que las dinámicas del laissez faire y del intervencionismo del Estado son necesariamente contradictorias e irreconciliables, esto es, porque los hechos han demostrado que la universidad puede estar subordinada simultáneamente a las demandas del entorno y a las reglas del Estado evaluador-orientador.

En todo caso, el punto crucial en el tránsito posible hacia la universidad heterónoma no es tanto qué margen de autonomía tienen las universidades para determinar su agenda proactivamente, sino cuáles son las consecuencias positivas y negativas de dicho tránsito para una sociedad éticamente participativa y comprometida con una educación superior no absurda, sino auténticamente existencial.

Sin duda encontramos procesos en nuestro país que, a nombre de la modernización y de la inserción de la universidad en el contexto de la sociedad del conocimiento, de los aprendizajes, de la informatización 
de la educación, han encontrado un terreno fértil para favorecer discursos claramente mercantiles sobre la Universidad.

Lamentablemente, las fuerzas sociales alternativas que han manejado y orientado algunas universidades en ciertos periodos históricos del Perú, han dejado espacios para que las perspectivas del 'capitalismo académico' ${ }^{10}$ tengan un desarrollo acelerado. Podría afirmarse que, las corrientes sociales alternativas, de centro izquierda, que en algún momento han tenido posibilidades de poder, no han concretado un proyecto universitario de corte social, ya sea por un exceso de ideologización o por la incapacidad de articular dinámicas sociales para la Universidad. (Cf. Coraggio, 2 001)

De tal modo, siguiendo a Levy nos encontramos en la tercera ola de la generación de las universidades, en donde la comercialización de los servicios educativos es factor eje de las políticas universitarias. De allí que, en los últimos tiempos se haya producido un crecimiento vertiginoso de universidades privadas, que buscan la absorción de la demanda educativa bajo la consigna: 'un ingresante por postulante'; o lo que es lo mismo, los que pueden pagar ingresan.

Más aún cuando existen, en los tiempos actuales, nuevas demandas sociales, que en la práctica operan relacionadas entre sí, y el significado o efectos de esas demandas involucran por antonomasia a la Universidad. Así han surgido nuevas demandas por estudios superiores en el contexto de la masificación educacional ${ }^{11}$, nuevas demandas por cambios en el contexto de información y conocimiento, nuevas demandas del contexto de desarrollo ${ }^{12}$, nuevas demandas del contexto cultural y nuevas demandas ocupacionales.

\section{POSIBILIDADES PARA DESARROLLO NACIONAL}

La universidad contemporánea el resultado, tanto de los procesos acumulativos de la problemática universitaria (de los imaginarios históricos), como de los desarrollos del contexto: globalización, crecimiento acelerado de las nuevas 'tecnologías de la información y comunicación' (TIC), transnacionalización de la política, profundización de la crisis social, masificación, diferenciación y expansión del sistema universitario. La universidad de hoy es la síntesis de los procesos históricos, políticos, sociales, económicos y culturales. Hablar de la universidad contemporánea es ,por supuesto, hablar de una totalidad. Cuando se quiere hablar en concreto es preciso hablar de la universidad peruana, en fin, de la concreción de ese discurso genérico que se denomina universidad contemporánea y que tiene, por supuesto, líneas muy claras que intentan convertirse en las líneas de concreción práctica en todos los contextos, esto significa, homogenizar el discurso sobre la Universidad.

Frente a esto, surge la necesidad insoslayable de plantear posibilidades que hagan frente a esta crisis existencial de la universidad peruana; propuestas enmarcadas dentro del diseño y ejecución de un "plan de desarrollo nacional a largo plazo" que contemple, a partir del ámbito educativo, una propuesta de desarrollo de la educación en la que la educación superior y, prioritariamente, la educación universitaria se sustente en la concepción de una universidad como entidad de altos estudios, portadora y productora de nuevos conocimientos científicos, entendida como un polo de desarrollo de potencial inimaginable.

Dentro de este contexto, se puede efectuar un planteamiento propositivo por el principio mutatis mutandis respecto de las variopintas cuestiones problemáticas de la realidad universitaria, las cuales se mencionan a continuación: 


\begin{tabular}{|c|c|}
\hline PROBLEMAS DE LA UNIVERSIDAD & PROPUESTAS DE SOLUCIÓN \\
\hline $\begin{array}{l}\text { a) Carencia de políticas educativas de Estado } \\
\text { que conduzcan al progreso sostenible del } \\
\text { sistema universitario. }\end{array}$ & $\begin{array}{l}\text { Creación de academias nacionales y consejos } \\
\text { interinstitucionales para sostener y articular proyectos } \\
\text { a largo plazo. }\end{array}$ \\
\hline $\begin{array}{l}\text { b) Heterogeneidad de concepciones y } \\
\text { segmentación universitaria que desconoce } \\
\text { las áreas prioritarias de investigación del } \\
\text { país. }\end{array}$ & $\begin{array}{l}\text { Establecimiento de sistemas nacionales de regulación } \\
\text { para la medición, evaluación, acreditación e } \\
\text { información pública de los estándares de calidad } \\
\text { científica y educativa que poseen las universidades del } \\
\text { país. }\end{array}$ \\
\hline 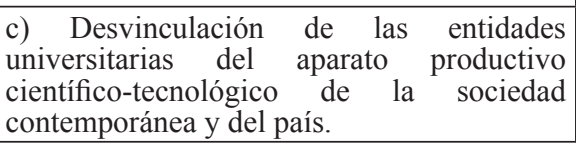 & $\begin{array}{l}\text { Establecimiento de convenios de colaboración } \\
\text { y alianzas estratégicas para articular procesos } \\
\text { de intercambio informativo y productivo entre } \\
\text { universidades locales y de otros países. }\end{array}$ \\
\hline $\begin{array}{l}\text { d) La masificación de la oferta educativa por } \\
\text { parte de las universidades en detrimento de la } \\
\text { calidad educativa. }\end{array}$ & $\begin{array}{l}\text { Creación de universidades de vanguardia en áreas } \\
\text { específicas de la ciencia en la que el país posea ventajas } \\
\text { comparativas y competitivas. Para este fin, se ha de } \\
\text { captar a los alumnos más talentosos del país, los cuales } \\
\text { han de ser formados por docentes investigadores de } \\
\text { primer nivel. }\end{array}$ \\
\hline $\begin{array}{l}\text { e) Inversión estatal masiva e igualitaria en el } \\
\text { sistema universitario. }\end{array}$ & $\begin{array}{l}\text { Privilegiar las inversiones en aquellas universidades } \\
\text { de vanguardia. }\end{array}$ \\
\hline $\begin{array}{l}\text { f) Docentes universitarios con escasas } \\
\text { calificaciones académicas y de investigación. }\end{array}$ & $\begin{array}{l}\text { Establecimiento de la carrera docente universitaria } \\
\text { meritocrática. }\end{array}$ \\
\hline $\begin{array}{l}\text { g) Reducción a la mínima expresión de } \\
\text { docentes con altas calificaciones académicas } \\
\text { y de investigación, debido a que la gestión en } \\
\text { las universidades están basadas en el imperio } \\
\text { de lo político, mas no de lo científico. }\end{array}$ & $\begin{array}{l}\text { Estimulación de la producción científica mediante la } \\
\text { constitución de un fondo económico intangible para el } \\
\text { desarrollo de proyectos de investigación de relevancia. }\end{array}$ \\
\hline h) Inexistencia de políticas de investigación. & $\begin{array}{l}\text { Establecer planes estratégicos y operativos de } \\
\text { desarrollo de la investigación con la participación de } \\
\text { los distintos estamentos de la sociedad. }\end{array}$ \\
\hline
\end{tabular}

\begin{tabular}{|c|c|}
\hline $\begin{array}{l}\text { PROBLEMAS DE LA } \\
\text { UNIVERSIDAD }\end{array}$ & PROPUESTAS DE SOLUCIÓN \\
\hline $\begin{array}{l}\text { i) Escasos recursos financieros } \\
\text { destinados para la investigación. }\end{array}$ & \begin{tabular}{lcccccc} 
Destino & de & una & partida & \multicolumn{2}{c}{ presupuestal no menos } \\
del $1 \%$ & de & PBI & exclusiva & para & la & investigación.
\end{tabular} \\
\hline 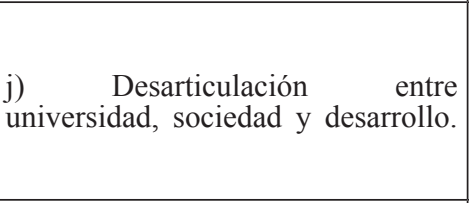 & $\begin{array}{l}\text { Desarrollo de la investigación en función de las ventajas } \\
\text { comparativas del país, de manera que esta repercuta en } \\
\text { la mejora de las condiciones de vida de la población. } \\
\text { Desarrollo por parte de las escuelas profesionales de } \\
\text { tecnología, aprovechando los conocimientos científicos } \\
\text { generados por las universidades de vanguardia. }\end{array}$ \\
\hline $\begin{array}{l}\text { k) La existencia de un modelo de } \\
\text { universidad anacrónico sustentado } \\
\text { en una legislación inoperante. }\end{array}$ & $\begin{array}{l}\text { Un cambio de modelo de universidad que se sustente en una } \\
\text { nueva ley universitaria que conciba a esta, a nivel nacional, como } \\
\text { un sistema, y a nivel específico, como una institución científica, } \\
\text { privilegiando la carrera académica en lugar de la carrera política. }\end{array}$ \\
\hline $\begin{array}{l}\text { 1) Trato igualitario entre r las } \\
\text { actividades administrativas } \\
\text { las } \quad \text { actividades } \quad \text { académicas. }\end{array}$ & $\begin{array}{l}\text { Establecimiento de linderos diferenciadores entre la labor } \\
\text { científica y la administrativa que permitan potenciar la capacidad } \\
\text { administrativa y la producción científica respectivamente. }\end{array}$ \\
\hline $\begin{array}{l}\text { 11) Excesivo poder de decisión } \\
\text { otorgado a los estudiantes en } \\
\text { el gobierno de la universidad. }\end{array}$ & $\begin{array}{l}\text { Otorgamiento a otros actores de la actividad universitaria del } \\
\text { poder de decisión en el gobierno universitario, así como los } \\
\text { estudiantes de postgrado y representantes de la sociedad civil. }\end{array}$ \\
\hline \begin{tabular}{lrrr|} 
m) & Mala & administración & de \\
los & recursos & financieros & de \\
la & universidad & e & inexistencia \\
de & rendición & de & cuentas.
\end{tabular} & $\begin{array}{l}\text { Establecer una administración financiera eficiente que sepa } \\
\text { dar rendición de cuenta de su gestión a la opinión pública. }\end{array}$ \\
\hline
\end{tabular}


Por lo visto, la calidad sigue siendo tema obligado de reflexión y debate cuando se trata de incentivar políticas que incentiven o incrementen desde el Estado y la sociedad el estándar científico de nuestras universidades. Una medida influyente de esta época es que es necesario crear un contexto de exigencias de cambio para instituciones universitarias estancadas, sin futuro y con un pasado desprestigiado que no sirve más como referente y seña de identidad. En tal sentido, se plantean las siguientes estrategias, las cuales se han de convertir en programas innovativos de Estado: a) Instalar la evaluación como práctica constante de las instituciones, los profesores y los alumnos; b) En la gestión de las instituciones, hay que evaluar más los resultados que los medios y los insumos, mostrando sus resultados a la sociedad; c) El Gobierno debe propiciar la competencia entre instituciones e impulsar la creación de nuevas instituciones tanto públicas como privadas; d) Someter la política laboral (contratación y promoción de académicos) a la evaluación, y vincular su salario a la calidad de su desempeño; e) Regular la admisión de estudiantes a las instituciones; f) Fortalecer el postgrado y la investigación; g) Establecer estímulos fiscales para el desarrollo tecnológico en empresas y su vinculación con las instituciones de educación superior; h) Profesionalizar la gestión de las instituciones, poniéndola en manos de personas moral y académicamente probadas.

En otros contextos, si escucháramos esto hoy en día, tal vez se diría “¡Pues qué triviales propuestas! ¿Acaso no se está sugiriendo lo que toda institución universitaria debería hacer de manera normal y cotidiana?". Al decir esto, se da cuenta de la distancia que nos separa de aquellas instituciones universitarias de otros países cercanos, pues, que duda cabe, estamos en momentos de grave crisis de la educación universitaria peruana.
Darnos cuenta de que la política de Estado ha consistido, en primer lugar, en poner a operar a las instituciones sobre una base elemental, mínima de decoro académico y administrativo. Recuperar la funcionalidad básica de las universidades es, en principio, el objetivo de la reforma de la educación superior universitaria que el Perú necesita.

\section{A MODO DE CONCLUSIÓN}

Hasta aquí se tiene los suficientes elementos de juicio para formular ideas a modo de conclusiones:

Primero, la Universidad, como institución que representa a la educación universitaria dentro de la sociedad, es en quien recae la responsabilidad del progreso de la ciencia y la tecnología de una nación. En ese sentido, se hace necesario una concepción de universidad que asuma el discurso humboldtiano de ser centro de investigación, y a partir del cual se construya un modelo universitario cuya diversificación se organice en forma de redes, que aproveche las potencialidades de las $\mathrm{TIC}^{13}$, posea sólidas bases disciplinarias, pero a la vez, sea inter y transdisciplinaria ${ }^{14}$ en muchos aspectos, que junto a la investigación académica acoja nuevos modos de producción de conocimientos, y que sea capaz de competir con los conglomerados universitarios de los países más desarrollados, a la vez de mantener presencia y arraigo local y regional.

De este modo, se puede afirmar que los modelos universitarios asociados con la centralidad investigativa se correlacionan con el progreso, y los demás con el fracaso. Un fracaso que supone atraso y dependencia cultural que cada vez se acentúa en nuestro país generalizando consecuentemente el nivel de pobreza existente. La universidad es el centro de fomento de la investigación y del cultivo del saber por excelencia. Universidad que no investiga es un cadáver en busca de una certificación mortuoria. 
Por ello, los obstáculos epistemológicos en la conceptualización de universidad han hipertrofiado, pongamos por caso, el peso de los currículos en detrimento del recurso humano y el conocimiento.

Segundo, no se ha de postergar decisiones presupuestarias hasta que dentro de varios años tengamos un plan estratégico. Es imprescindible invertir recursos ya, para crear condiciones iniciales que permitan abrir el espacio y ganar el tiempo para esa revisión y planificación estratégica. Ello permitiría salir de este juego de suma cero cortoplacista, donde los corporativismos, faccionalismos partidarios y clientelismos universitarios de diverso tipo se fortalecen en una lucha por la sobrevivencia que sustituye la reflexión sobre los derechos de los ciudadanos, el proyecto de sociedad y país, y el papel de la educación, la ciencia, la tecnología y las artes para lograrlos. Esos recursos existen, y deberían ser tomados como anticipo del desarrollo futuro, imponiendo al sector monopólico rentista, o a los sectores que hoy los evaden o eluden, el pago de impuestos que contribuyan al desarrollo de un país del que han extraído ganancias extraordinarias a nivel mundial sin comprometerse con su desarrollo, gobernabilidad y sostenibilidad a futuro.

Esta renovación del sistema de educación, ciencia y técnica, clave para el paradigma científico-tecnológico y para la sociedad centrada en el conocimiento y la información, no podría hacerse, sino como parte de una estrategia de rearticulación y dinamización del contexto interno, para que el Perú retome el rumbo del desarrollo, pieza ausente del fiscalismo economicista predominante.

En consecuencia, la política educativa no puede ser pensada independientemente de la política económica y de las políticas sociales, ni la educación puede ser pensada independientemente de la economía y de la sociedad. Un modelo económico puede subordinarla y mercantilizarla, como en el caso del neoliberalismo, o autonomizarla y valorarla como bien público, condición esencial de su propio desarrollo, como han hecho y hacen los países que hoy pueden competir como iguales en el mundo.

Tercero, hay dos buenas razones para reformar la educación universitaria del siglo XXI: una económica y otra personal. El muy ensayado argumento económico nos dice que el conocimiento está cambiando tan rápidamente que no podremos proporcionar a los jóvenes la base que necesitarán, sencillamente, porque desconocemos cuál será esa base. Por ello, debemos ayudarles a desarrollar una mente dúctil y ágil, de manera que puedan aprender aquello que les sea necesario. Si conseguimos esto, tendremos una fuerza trabajadora mundial en la que habrá personas innovadoras y dotadas de recursos. El argumento personal lleva a esta misma conclusión. Muchos jóvenes se sienten claramente inútiles ante todas las incertidumbres y complejidades lo que Dahrendorf llama el "reino de anomia" del mundo contemporáneo.

Por tanto, no esperemos que aquella idea sostenida por José Ortega y Gasset, que es más fácil cambiar un cementerio de lugar que hacer que las universidades cambien, sea verdad.

\section{REFERENCIAS BIBLIOGRÁFICAS}

APPLEBERRY, J. J. 1998. citado por Vidal Sunción Infante. (1999) "O Perfil da Universidade para o Próximo Milenio", Education Policy Análisis Archive, Vol. 7, Number 32. Disponible en http://epaa. asu.edu/epaa/v 7 n32/

ARIAS C., S. y E. MOLINA (2008) Universidad y cooperación al desarrollo. La experiencia de las universidades de Madrid. Edición Madrid: Catarata

BANCO MUNDIAL. (2000). La educación superior en los países en desarrollo: peligro y promesas. Washington: El Banco.

BONVECCHIO, C. (2000) El mito de la 
universidad. (11 ed.). México: Siglo Veintiuno.

BRUNNER, J. J. (2 000) "Innovación en las políticas y políticas de innovación" en Actas del V Seminario Internacional de Educación Superior. Políticas de Educación Superior: ¿Tiempo de Innovar? Consejo de Educación Superior de Chile (CSE)

CLARK, B. R. (1996) El problema de la complejidad en la educación superior moderna. En: Rothblatt, S. y Wittrock, B. (Comps.). La universidad europea $y$ americana desde 1800. Las tres transformaciones de la Universidad moderna. Barcelona: Pomares-Corredor. (Educación y Conocimiento).

(1998) Creating

Entrepreneurial

Organizational

Transformation. Guildford, Surrey: Pergamon-IAU Press.

CORAGGIO, J. L. (2001) Construir universidad en la adversidad. Desafios de la educación superior en América Latina. Washington: OEA/OAS.

DABAT, A. (2 009) Globalización, conocimiento y desarrollo: Teoría y estrategias de desarrollo en el contexto del cambio histórico mundial. México D.F. : Universidad Nacional Autónoma de México, Instituto de Investigaciones Económicas

DÍAZ-AGUADO, M. y C. MEDRANO (2 000) Educación y Razonamiento Moral. Bilbao: Ediciones Mensajero, S.A.

DIDRIKSSON, A. (2 000) La universidad de la innovación. Una estrategia de transformación para la construcción de universidades de futuro. México: IESALC/UNESCO y Gobierno del Estado de Zacatecas.

FILMUS, D. (2 000) "Educación y desigualdad en América Latina de los '90. ¿Una nueva década perdida?’. En
Tünnermann, C. y López, F. (Coords.). La educación en el horizonte del siglo $X X I$. p. 17-52. Caracas: IESALC/ UNESCO.

GANDARILLA S., J. (comp.) (2 007) Reestructuración de la universidad y del conocimiento. México D. F.: Centro de Investigaciones Interdisciplinarias en Ciencias y Humanidades

GARDNER, H. (1 999) The disciplined mind. What all students should understand, New York: Simon \& Schuster.

GIBBONS, M. (1 998) Pertinencia de la educación superior en el siglo XXI. Washington: Banco Mundial.

GONZÁLEZ, P. (2 001) La universidad necesaria en el siglo XXI. México: Era. (Colección Problemas de México).

HUMBOLDT, W. V. (2 000) La situación de la universidad. En Bonvecchio, C. El mito de la universidad. (11 ed.). México: Siglo Veintiuno.

LEVY, D. (1 995) La Educación Superior y el Estado en Latinoamérica. Desafios privados al predominio público. México: Ed. UNAM.

MICHAVILA, F. (2 005) «Cinco ideas innovadoras para la europeización de la educación superior» [artículo en línea]. Revista de Universidad y Sociedad del Conocimiento (RUSC). Vol. 2, n. ${ }^{\circ}$ 1. UOC. [Fecha de consulta: $\mathrm{dd} / \mathrm{mm} /$ aa]. $\quad<$ http://www.uoc.edu/rusc/dt/esp/ michavila0405.pdf $>$

PISCOYA H., L. (2 004) "Un Currículo para la antieducación". En Revista de la Facultad de Educación de la Universidad Nacional Mayor de San Marcos, año I, $\mathrm{N}^{\mathrm{o}} 1$.

RIBEIRO, D. (1 971) As Américas e a Civilização - Processo de Formação e Causas do Desenvolvimento Cultural Desigual dos Povos Americanos. Edição brasileira $\left(1^{\circ}\right)$, Rio de Janeiro: Editora Civilização Brasileira. En versión española Las Américas y la Civilización. 
Proceso de Formación y causas del desarrollo desigual de los pueblos americanos. (trad.) (1985) Bs. As.: Bibliotecas Universitarias.

SALMI, J. (2 000) Higher Education at a Turning Point. (manuscrito)

SCHWARZMAN, S. (2 001) "La universidad como empresa económica”. En Revista de la Educación Superior, XXX (1), 117.

YARZÁBAL, L. (1 999) Consenso para el cambio en la educación superior. (1 ed.). Caracas: IESALC/UNESCO.

\section{NOTA}

1 Significa quebrar el 'mito del sobrediagnóstico' de la realidad educacional universitaria. La creencia de que en el Perú ya se han realizado excesivos diagnósticos de la situación de nuestro sistema universitario $y$, consecuentemente, ya sabemos al respecto más que suficiente y lo que se necesita prioritariamente es no continuar perdiendo el tiempo en más estudios para dedicarse a la producción de resultados. No obstante, este mito repetido solemnemente y de modo altisonante no resiste el menor análisis. ¿Puede afirmarse seriamente que sabemos más de lo necesario de nuestro sistema universitario cuando no existe un registro nacional de producción investigativa por universidades, por áreas científicas; cuando no existe informe sobre la calidad institucional, administrativa y académica ni un ranking de universidades del Perú? (Cf. Piscoya, 2004).

2 En el 'modelo de la universidad colonial', existió la pretensión de autogobernarse (antecedente importante de la autonomía universitaria), de la cual la universidad de este periodo jamás llegó a disfrutar plenamente, porque las carreras que se ofrecían tenían vínculos directos con los intereses monárquicos. De igual forma, existió un antecedente de la participación estudiantil que se traducía en el derecho a votar en el discernimiento de las cátedras (primer antecedente de cogestión universitaria). Estos elementos constituyen una de las características fundamentales de la universidad latinoamericana de la etapa colonial.

3 El modelo de universidad de Bolonia (universitas scholarium) surgió de los ímpetus estudiantiles que buscaban profesores; ellos participaban directamente en el gobierno y administración universitaria. El prestigio de Irnerius, como primer glosador o comentarista del Código de Justiniano permite que la fama de Bolonia atraiga muchos estudiantes extranjeros. Luego, Gratianus funda la ciencia del Derecho Canónico que contribuyó grandemente a acrecentar el prestigio de esta universidad. Bolonia, aunque parezca raro, llegó a poseer en sus aulas hasta diez mil estudiantes en el siglo XII. Dante y Petrarca la visitaban frecuentemente. En esta institución para ser Rector-estudiante había que ser clérigo, tener 24 años de edad, estudiar quinto año y vestir traje talar.

4 La universidad de París (universitas magistrorum), llamada la Gran Universidad Teológica, nacida para prestar servicio a las necesidades de la Iglesia Católica era gobernada por los profesores, que formaron una corporación que luchó para defender su autonomía ante el canciller y la autoridad civil. Al Papa se recurría en caso de litigio. En esta época, se practicaba la libertad de cátedra debido al auge de las discusiones y de la lógica, método impulsado por Pedro Abelardo, eminente Maestro de la universidad, pero las autoridades de la Iglesia Católica presintieron el peligro de esta 
práctica en contra de sus intereses. No está de más decir que la Universidad de París recibió la protección de las autoridades eclesiásticas, ya que era la fortaleza de fe y de la ortodoxia católica. Por eso, la vigilaban estrictamente y supervisaban su enseñanza y actuación para evitar cualquier desviación.

5 El 'modelo napoleónico' implantó la extirpación autoritaria del pensamiento en la universidad, favoreciendo la enseñanza de los profesores y de las ciencias aplicadas, pero no de las ciencias básicas. Este modelo fue el que inspiró a las universidades latinoamericanas del siglo XIX; fue el patrón francés de universidad, aunque, en realidad, no era otra cosa que un conglomerado de escuelas autárquicas. (Cf. Ribeiro, 1971: 70)

6 En 1918, los estudiantes de la Universidad Nacional de Córdoba hastiados de la labor anticientífica de sus academias, del horror al progreso y a la cultura, de la ineptitud de sus dirigentes, del estancamiento e inmoralidad reinante, llevan a cabo un movimiento reivindicativo que tendría, posteriormente, gran resonancia en las universidades de toda Latinoamérica. Los factores situacionales que desencadenaron esta reforma lo constituyen el carácter elitista de la formación, el énfasis profesionalista, la estructura académica construida sobre una simple federación de facultades o escuelas profesionales semiautomáticas, la organización tubular de los profesores (escasa posibilidad de transferencia de un currículo a otro; rígidos y que provocaban la duplicidad de personal docente, equipos, bibliotecas, etc.) carrera docente incipiente (los docentes con pocas horas dedicadas a tareas de enseñanza, inclusive aquellos que tenían nombramiento de tiempo completo), la ausencia de una organización administrativa eficaz (poca atención a la administración académica y a la administración de la ciencia), métodos docentes basados principalmente en la cátedra y la simple transmisión del conocimiento (deficiente enseñanza práctica por limitaciones en cuanto a equipos, bibliotecas y laboratorios), proyección limitada por la escasez de recursos que se destinan a atender las labores docentes, la inexistencia de vínculos con la comunidad local y nacional ni con el sector productivo.

7 El principal inspirador del modelo alemán de Universidad fue Wilhelm Von Humboldt, el cual fundó en 1810 la Universidad de Berlín, hoy conocida como la Universidad Humboldt. Concibió y organizó la universidad como una institución dedicada a la investigación y a la formación de científicos.

8 Otro modelo de universidad que surge en el siglo XX es el de la 'multiversidad norteamericana' cuyo postulado eje es la democratización de la universidad, dotándole a esta su papel social y a la vez, manteniendo el rigor en la investigación científica universitaria. Una concepción que pretende correlacionar universidad y sector productivo, también llamado 'modelo de universidad empresa'. Este modelo para muchos constituye una segunda revolución académica debido a la contribución directa al crecimiento económico de los países gracias a la labor de sus universidades.

$9 \mathrm{El}$ 'entorno' es entendido como el conjunto de factores externos a la universidad que ejercen influencia directa en la institución universitaria. Así podemos señalar la existencia de un entorno general conformado por las demandas políticas, sociales, económicas, culturales-religiosos, científico- 
tecnológicas, educativas, geográficas y las tendencias de desarrollo en el ámbito internacional, continental, nacional y local. Un entorno específico conformado por las otras instituciones locales: institutos, academias y consejos científicos.

10 Se entiende por 'capitalismo académico' a aquellos recursos financieros provenientes de sectores gubernamentales estatales y/o privados que condicionan y privilegian hacia ciertos objetivos sociales particulares el avance técnico y científico (filosofía colectivista de universidad). Por otro lado, también se refiere a la concepción del conocimiento como 'mercancía' o 'cosificación' cuyo patrimonio pertenece al mejor postor (filosofía utilitarista de universidad).

11 El fenómeno de la 'masificación universitaria' (segunda ola) se inicia en la década del setenta en pleno siglo $\mathrm{XX}$. Este crecimiento expansivo se da en dos ámbitos. El primero se refiere a la creación de nuevas universidades, y el segundo, al acceso masivo de estudiantes de las clases medias y bajas, lo cual hizo que la universidad deje de ser elitista. (Cf. Levy, 1995)

12 Se entiende por 'desarrollo' al cambio cualitativo antes que al cuantitativo, pues este último es un factor condicional necesario aunque no suficiente para el desarrollo. Ello es producido al mejorar todas las dimensiones de la entidad universitaria, controlando lo mejor posible las variables perturbadoras. Por tanto, no es una simple renovación o una innovación parcial; antes bien, es un mejoramiento de conjunto (sistémico) y continuo.

13 La integración educacional de los medios electrónicos y los recursos virtuales amplían el acceso del material de calidad a una mayor red social; sin embargo, requiere, además del diseño científicotécnico, un diseño sociocultural para que los principios y valores de nuestra sociedad sobrevivan en el ambiente multimedial a través de una interacción creativa maestro-alumno y alumnoalumno. De este modo, se afrontaría, positivamente, la tensión existente entre los medios digitalizados y los valores sociales. (Cf. Gandarilla, 2007:91)

14 La investigación intercurrente e interdisciplinaria es una necesidad insoslayable para el desarrollo científico y el desarrollo social. Aún más, se requiere una investigación en cooperación interinstitucional para el desarrollo, cuyo propósito sea ampliar los campos de conocimiento. (Cf. Arias y Molina, 2008: 327; Dabat, 2009: 264). 